\title{
Estudio AB-Initio de las propiedades estructurales y electrónicas de la doble Perovskita $\mathrm{Ba}_{2} \mathrm{InTaO}_{6}$
}

\author{
\begin{tabular}{l|l|l|l} 
Crispulo Enrique Deluque-Toro & David Arsenio Landinez-Tellez & Jairo Arbey Rodríguez-Martínez & \\
& & Jairo Roa-Rojas
\end{tabular}
}

Recibido:

Diciembre 10 de 2012

Aceptado:

Abril 25 de 2013

'M.Sc en Física, Grupo de Nuevos Materiales, Universidad Popular del Cesar, Valledupar, Colombia. Correo electrónico: deluquetoro@gmail.com

${ }^{2} \mathrm{Ph} . \mathrm{D}$ en Física, Gema: Grupo de Estudio de Materiales,

Departamento de Física, Universidad Nacional de Colombia, Bogotá, Colombia. Correo electrónico: dalandinezt@unal.edu.co

${ }^{3}$ Ph.D En Física, Gema: Grupo de Estudio de Materiales, Departamento de Física, Universidad Nacional de Colombia, Bogotá, Colombia. Correo electrónico: jarodriguezm@bt.unal.edu.co

${ }^{4} \mathrm{Ph}$.D Física, Grupo de Física de Nuevos Materiales, Departamento de Física, Universidad Nacional de Colombia, Bogotá, Colombia. Correo electrónico: jroar@unal.edu.co

\section{Resumen}

Los materiales de tipo Perovskita doble con fórmula genérica $\mathrm{A}_{2} \mathrm{BB}^{\prime} \mathrm{O}_{6}$ han sido ampliamente estudiados en los últimos años debido a su gran versatilidad, la cual permite la inclusión de iones de tipo alcalino térreo en los sitios A de la estructura y metales de transición en los sitios $B$ y B', dando origen a diversas propiedades físicas que redundan en múltiples aplicaciones industriales. En este trabajo estudiamos las propiedades estructurales y electrónicas del compuesto $\mathrm{Ba}_{2} \mathrm{In} \mathrm{TaO}_{6}$, el cual es de particular interés ya que dichos sistemas podrían aplicarse en el desarrollo de resonadores dieléctricos y filtros para señal de microondas en teléfonos móviles y otros dispositivos inalámbricos, entre otras. Mediante modelamiento computacional ab-initio, basado en la Teoría de Funcional Densidad (DFT), y partiendo de la estructura de grupo espacial Fm-3m, evaluamos los parámetros de red, las energías de equilibrio y la ecuación de estado entre otras cantidades. La minimización de la energía en función del volumen permite la obtención de un parámetro de red de 15.861 Bohr. Los resultados de la Densidad de Estados Electrónicos (DOS) muestran que la doble Perovskita $\mathrm{Ba}_{2} \mathrm{InTa \textrm {O } _ { 6 }}$ tiene un gap indirecto de $\sim 4.25 \mathrm{eV}$.

Palabras clave: Doble Perovskita, AB-Initio, estructura electrónica.

\section{Abstract}

The double Perovskites materials with the formula $\mathrm{A}_{2} \mathrm{BB}^{\prime} \mathrm{O}_{6}$ has been studied thoroughly in the past years due to its versatility, which allow to include the alkaline earth ions in A sites and transition metals in B and B'sites, giving origin to several physical properties with various industrials applications. In this work the structural and electronic properties of $\mathrm{Ba}_{2} \mathrm{InTaO}_{6}$ are studied, material that is particularly interesting due to its possible application to the development of dielectric resonators and microwave signal filters on mobile phones and other wireless devices. Using ab-initio computational model, based on the Density Funtional Theory (DFT), and starting from spacial group Fm-3m, we calculate lattice parameters, equilibrium energies and equations of state among other quantities. The energy minimization as a function of volume allows to obtain a 
No. 1

Enero-Junio 2013 ISSN $0122-820 \mathrm{X}$ lattice constant of 15.861 Bohr. The results of electronic density of states (DOS) show that the double Perovskite $\mathrm{Ba}_{2} \mathrm{InTaO}$ has an indirect gap of $\sim 4.25 \mathrm{eV}$.

Keywords: Double Perouskites, $\mathrm{Ba}_{2} \operatorname{InTaO} \mathrm{O}_{6}$ DFT, Electronic structure.

\section{Introducción}

$\mathrm{E}$ compuesto $\mathrm{Ba}_{2} \mathrm{InTaO}_{6}$ es un material tipo perovskita doble de fórmula genérica $\mathrm{A}_{2} \mathrm{BB}^{\prime} \mathrm{O}_{6}$, con múltiples aplicaciones industriales [3-6], específicamente, en resonadores dieléctricos y filtros para señal de microondas en teléfonos móviles y otros dispositivos inalámbricos [7,8]. Aunque se han realizado diversos estudios experimentales sobre la estructura del compuesto $\mathrm{Ba}_{2} \mathrm{InTaO}_{6}$, su verdadera estructura cristalina no ha sido esclarecida aun. Dias et al. [1] reportan que el compuesto $\mathrm{Ba}_{2} \mathrm{InTaO}_{6}$ presenta una estructura tetragonal a bajas temperaturas, estos resultados fueron obtenidos mediante la técnica Espectroscopía Raman; sin embargo, esta afirmación fue refutada posteriormente por T.S. Hammink et al. [2], utilizando la técnica de difracción de rayos $\mathrm{X}$, aseguran que el compuesto presenta una estructura cúbica con grupo espacial Fm-3m en el rango de temperaturas 82-723 K. Además, no existen estudios basados en la DFT, a cerca de las propiedades estructurales y electrónicas del compuesto $\mathrm{Ba}_{2} \mathrm{InTaO}_{6}$. En el presente trabajo presentamos un estudio detallado de las propiedades estructurales y electrónicas del compuesto $\mathrm{Ba}_{2} \mathrm{InTaO}_{6}$ en el grupo espacial Fm-3m.

\section{Materiales y métodos}

Los cálculos fueron realizados utilizando la teoría de la funcional densidad (DFT) [9] implementada según el código Wien2k [10]. Las energías de correlación e intercambio fueron evaluadas en la aproximación de gradiente generalizado (GGA) según los potenciales propuestos por Perdew-Burke-
Ernzerhof-96 [11]. Los radios de muffin-tin (en u.a.) fueron 2.3, 1.7, 1.75 y 1.6 para el Ba, In, Ta y $\mathrm{O}$ respectivamente, el $\mathrm{R}_{\mathrm{MT}} * \mathrm{~K}_{\text {max }}=7.0$, el máximo valor del momento angular fue 10. $G_{\text {máx }}$ que es el máximo vector del espacio recíproco para el desarrollo del potencial en serie fue de 12.0. Se tomó una malla de 3000 puntos $\mathrm{k}$ que equivale a un máximo de 104 puntos k en la zona irreducible de Brillouin.

El criterio de convergencia para el cálculo autoconsistente de las energías totales es de $0.0001 \mathrm{Ry}$ en la energía y 0.0001 u.a. en la carga. El cálculo se efectúo teniendo en cuenta las relajaciones internas de los átomos hasta que las fuerzas fueron inferiores a $1 \mathrm{mRy}$ /Angstrom. Así mismo, todos los cálculos fueron realizados sin considerar la polarización de spin, debido a la baja característica magnética de los cationes de In y Ta.

\section{Resultados y discusión}

En la Figura 1 se presenta la estructura cúbica de la Perovskita $\mathrm{Ba}_{2} \mathrm{InTaO}_{6}$ estudiada en este trabajo.

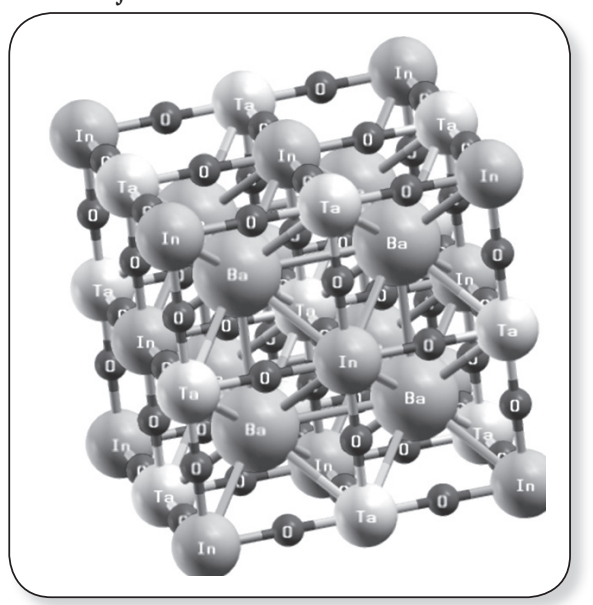

Figura1. Estructura de la perovskita $\mathrm{Ba}_{2} \mathrm{InTaO}_{6}$ 
En la Figura 2 se presentan los resultados de la energía en función del volumen para la estructura cúbica del compuesto $\mathrm{Ba}_{2} \mathrm{InTaO}_{6}$. En la gráfica se observa que existe un mínimo de energía y que por tanto el material es estable o metaestable energéticamente.

El valor del volumen en el mínimo de energía corresponde al volumen de equilibrio, y de allí se deduce la constante de red. Estos resultados fueron ajustados mediante la ecuación de estado Murnaghan [12]. El valor mínimo de energía obtenido es -76480.298 $\mathrm{eV}$, el volumen de equilibrio encontrado es 997.616 Bohr ${ }^{3}$, la constante de red es $a=15.861$ Bohr, y el módulo de volumen obtenido es de $\mathrm{Bo}=155.7 \mathrm{GPa}$ y su derivada respecto a la presión es $B o^{\prime}=5.0$. El parámetro de red obtenido tiene buen acuerdo con los resultados experimentales [2] con un margen de error $\sim 1.4 \%$.

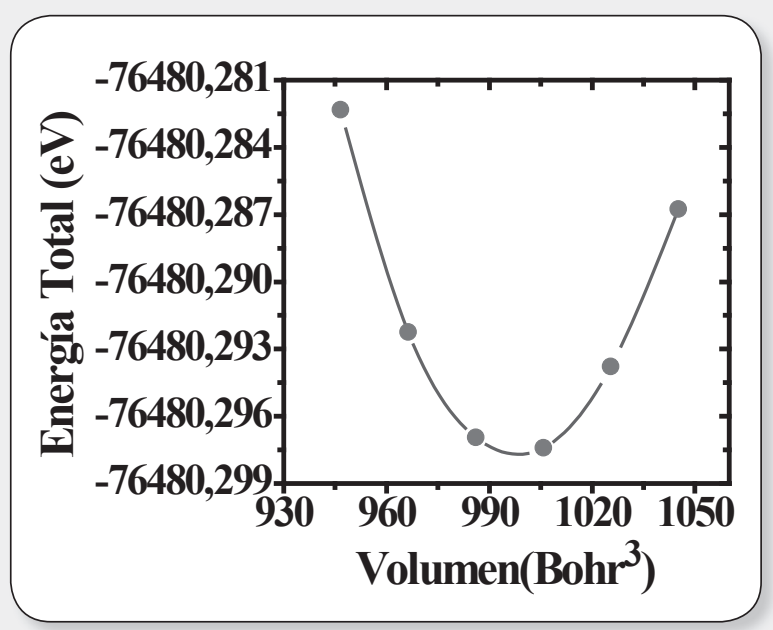

Figura 2. Energía total en función del volumen para el compuesto $\mathrm{Ba}_{2} \mathrm{InTaO}_{6}$.

La Tabla 1 muestra las posiciones atómicas en la Perovskita $\mathrm{Ba}_{2} \mathrm{InTaO}_{6}$, obtenidos por la optimización de las coordenadas internas. Los resultados están en buen acuerdo con los obtenidos de los experimentos [2].
Tabla 1. Resultados de las posiciones internas de la Perovskita $\mathrm{Ba}_{2} \mathrm{InTaO}_{6}$, obtenidas a partir de nuestros cálculos ab-initio. Los datos experimentales se incluyen para la comparación.

\begin{tabular}{lccc}
\hline Perovskita & Sitios & GGA: $(\boldsymbol{x}, \boldsymbol{y}, \boldsymbol{z})$ & Exp.: $(\boldsymbol{x}, \boldsymbol{y}, \boldsymbol{z})$ \\
\hline & Ba: $8 c$ & $0.25,0.25,0.25$ & $(0.25,0.25,0.25)^{\mathrm{a}}$ \\
Ba $_{2}$ InTaO $_{6}$ & In: $4 a$ & $0,0,0$ & $(0,0,0)^{\mathrm{a}}$ \\
& Ta: $4 b$ & $0.5,0.5,0.5$ & $(0.5,0.5,0.5)^{\mathrm{a}}$ \\
& O: $24 e$ & $0.2607,0,0$ & $(0.2568,0,0)^{\mathrm{a}}$ \\
\hline
\end{tabular}

${ }^{a}$ Exp. [2].

En la Figura 3 se presentan las propiedades electrónicas de la Perovskita $\mathrm{Ba}_{2} \mathrm{InTaO}_{6}$, el estudio se realizó mediante la DOS en función de la energía y la estructura de bandas a lo largo de algunas direcciones de alta simetría de la primera zona de Brillouin; en esta figura se observa la energía de los electrones en función del vector de onda $k$, tomado a lo largo de las direcciones W-L- $\Lambda-\Gamma-\Delta-\mathrm{X}-\mathrm{Z}-\mathrm{W}$.

La DOS y la estructura de bandas se calculan para la configuración en equilibrio usando la constante de red correspondiente al volumen que minimiza la energía; el cero de la energía se ha elegido en el nivel de Fermi. Se observa que el compuesto $\mathrm{Ba}_{2} \mathrm{InTaO}$, presenta un comportamiento aislante debido a que tiene un gap indirecto de aproximadamente $4.25 \mathrm{eV}$, en el nivel de Fermi. Además, en la Figura 3 se observa que existen cinco regiones principales: la primera se localiza debajo de $\sim-16 \mathrm{eV}$, formada principalmente por electrones $2 \mathrm{~s}-\mathrm{O}$ y una pequeña contribución 6s-Ta. Una segunda región bien definida entre -12 y $-13 \mathrm{eV}$, formada por los electrones $4 \mathrm{~d}$ In. La tercera región se encuentra localizada por debajo $-10 \mathrm{eV}$, formada por $6 \mathrm{~s}-\mathrm{Ba}$. Una cuarta región entre $\sim-6.0 \mathrm{eV}$ y el nivel de Fermi formada principalmente por electrones $\mathrm{p}-\mathrm{O}, 5 \mathrm{~d}-\mathrm{Ta}$ y pequeña contribución de $5 \mathrm{~s}$ In, $\mathrm{p}$-In y $6 \mathrm{~s}-\mathrm{Ta}$ y una quinta región ubicada $\sim 4.25 \mathrm{eV}$ hasta $8.8 \mathrm{eV}$ formada principalmente por los electrones $5 \mathrm{~d}$-Ta y unas contribuciones minoritarias de los electrones $5 \mathrm{~s}$-In, 6s-Ba, p del In y el $\mathrm{O}$.
Enero-Junio 2013 ISSN 0122-820X 


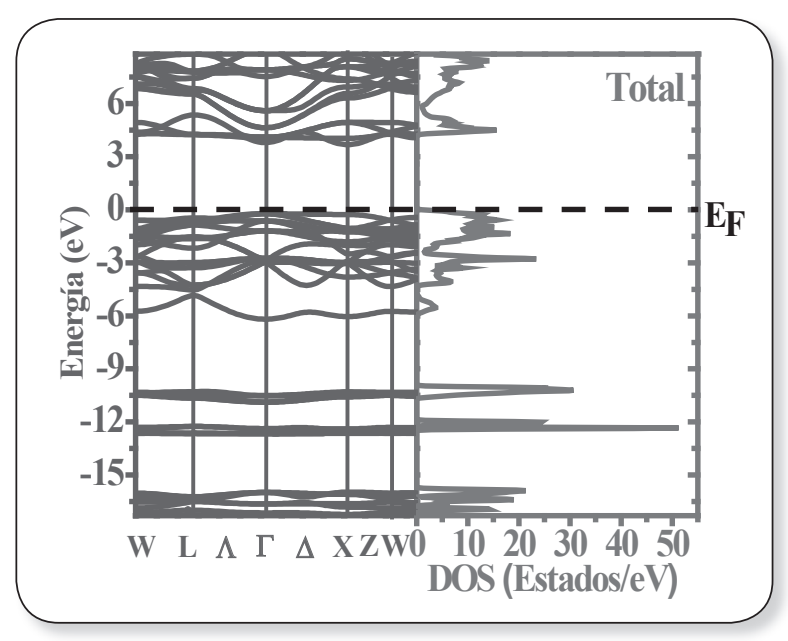

Figura 3. Estructura de banda y DOS para $\mathrm{Ba}_{2} \mathrm{InTaO}_{6}$ en la fase cúbica en condiciones de mínima energía.

\section{Conclusiones}

Utilizando DFT se calcularon las propiedades estructurales y electrónicas del compuesto $\mathrm{Ba}_{2} \mathrm{InTaO}_{6}$ en la estructura cúbica usando el método de Ondas Planas Aumentadas y Linealizadas (FP-LAPW) tal como está implementado en el código wien2k. Utilizando la gráfica de energía en función del volumen se determinaron algunas características estructurales como son la energía total, volumen de equilibrio, módulo de volumen y el parámetro de red que minimiza la energía. Además, se puede concluir que el material exhibe un comportamiento aislante debido a que presenta un gap indirecto de aproximadamente $4.25 \mathrm{eV}$.
[4] V. Ting, Y. Liu, R.L. Withers, E. Krausz, J. Solid State Chem. 177 (2004) 979_ 986.

[5] V. Ting, Y. Liu, R.L. Withers, L. Noren, M. James, J.D. Fitz Gerald, J. Solid State Chem. 179 (2006) 551-562.

[6] W.T. Fu, D.J.W. Ijdo, Solid State Commun. 134 (2005) 177-181.

[7] W. Wersing, Curr. Opin. Solid State Mater. Sci. 1 (1996) 715-731.

[8] I.M. Reaney, D.M. Iddles, J. Am. Ceram. Soc. 89 (2006) 2063-2072.

[9] P. Hohenberg, and W. Kohn, Phys. Rev. 136 (1964) 864.

[10] P. Blaha, K. Schwarz, G.K.H. Madsen, D. Kvasnicka y J. Luitz, WIEN2k_10.1, Techn. Universitat Wien, Austria, 2001.

[11] J.P. Perdew, S. Burke and M. Ernzerhof, Phys.Rev.Let. 77 (1996) 3865.

[12] F. D. Murnaghan, Proc. Natl. Acad. Sci., USA. 30 (1944) 244.

[1] A. Dias, L.A. Khalam, M.T. Sebastian, R.L. Moreira, J. Solid State Chem. 180 (2007) 2143-2148.

[2] T.S. Hammink, W.T. Fu, D.J.W.I.Jdo, J. Solid State Chem. 184 (2011) 848-851

[3] T.A. Vanderah, Science 298 (2002) 1182-1184. 